\title{
Application of the SAMINT methodology to the new cross section evaluations of ${ }^{63} \mathrm{Cu}$ and ${ }^{65} \mathrm{Cu}^{*}$
}

\author{
Vladimir Sobes ${ }^{1, a}$ and Luiz Leal ${ }^{2}$ \\ 1 Oak Ridge National Laboratory, Reactor and Nuclear Systems Division, Oak Ridge, TN, USA \\ 2 Institute for Radiological Protection and Nuclear Safety, Neutronics and Criticality Safety Assessment Department, Fontenay aux \\ Roses, France
}

\begin{abstract}
The SAMINT methodology allows coupling of differential and integral data evaluations in a continuous-energy framework. Prior to development of the SAMINT code, integral experimental data such as in the International Criticality Safety Benchmark Experiments Project remained a tool for validation of completed nuclear data evaluations. Now, SAMINT extracts information from integral benchmarks in the form of calculated sensitivity coefficients by Monte Carlo codes such as CE TSUNAMI-3D or MCNP6 and combines it with the results of experimental cross section measurements to produce an updated cross section evaluation utilizing information from both sets of data. The use of the generalized linear least squares methodology ensures that proper weight is given to both the differential and integral data. SAMINT is not intended to bias nuclear data toward specific integral experiments, but it should be used to supplement evaluation of differential experimental data. This work demonstrates the application of the SAMINT methodology to the new Oak Ridge National Laboratory (ORNL) evaluations of the resonance parameters for two isotopes of copper: ${ }^{63} \mathrm{Cu}$ and ${ }^{65} \mathrm{Cu}$.
\end{abstract}

\section{Introduction}

The SAMINT methodology allows for the coupling of differential and integral data evaluation in a continuousenergy framework [1]. More specifically, SAMINT was developed to allow the nuclear data evaluator to update the parameters of a resolved resonance region evaluation based on information from integral experiments, just as is traditionally done for differential cross section measurements. The computer code SAMINT works together with the mathematical machinery of the evaluation code SAMMY [2] to update resolved resonance parameters based on integral data.

Traditionally, SAMMY has used differential experimental data-i.e., $\sigma\left(E_{i}\right)$ vs. $E_{i}$ - to adjust nuclear data parameters such as resonance energies, resonance widths, the number of prompt neutrons emitted per fission, etc. Integral experimental data such as the International Criticality Safety Experiments Project (ICSBEP) benchmarks [3] have remained only a tool for validation of completed

\footnotetext{
a e-mail: sobesv@ornl.gov

${ }^{*}$ This manuscript has been authored by UT-Battelle, LLC, under Contract No. DE-AC0500OR22725 with the US Department of Energy. The US Government retains and the publisher, by accepting the article for publication, acknowledges that the US Government retains a nonexclusive, paid-up, irrevocable, worldwide license to publish or reproduce the published form of this manuscript, or allow other to do so, for the US Government purposes. The Department of Energy will provide public access to these results of federally sponsored research in accordance with the DOE Public Access Plan (http:// energy.gov/downloads/doe-public-access-plan).
}

nuclear data evaluations. SAMINT can be used to extract information from integral benchmarks to aid the nuclear data evaluation process. Near the end of the evaluation based on differential experimental data, integral data can be used alongside the differential data to resolve remaining ambiguity between differential data sets, guide the evaluator to troublesome energy regions, and inform the evaluator of the nuclear data parameters most important for integral benchmark calculations.

SAMINT is not intended to bias nuclear data towards fitting a certain set of integral experiments; rather, it should be used to supplement evaluation of differential experimental data. Using the generalized linear leastsquares (GLLS) methodology ensures that proper weight is given to the differential data.

As the first demonstration of the SAMINT capability, the resonance widths for resonances above the threshold of the first inelastic channel were adjusted for the resolved resonance region evaluation of ${ }^{56} \mathrm{Fe}$ based on the differential cross section measurements and four integral benchmarks from the ICSBEP handbook [3]. This work focuses on the application of the SAMINT methodology to the resolved resonance region evaluation of the two isotopes of copper. In contrast to the work discussed in Reference 1, this work focuses on highlighting the application of the SAMINT methodology to the case where many integral experiments are available and systematically treats the case of integral experiments that suggest conflicting changes. Important implications of the effect of the resonance parameter adjustment on integral experiments not considered in the SAMINT analysis are also discussed. 
Table 1. Differential experimental data used in the ${ }^{63} \mathrm{Cu}$ and ${ }^{65} \mathrm{Cu}$ resolved resonance region evaluations.

\begin{tabular}{|c|c|c|c|}
\hline Reference & Energy & Facility & Measurement \\
\hline Pandey et al. [5] & $32 \mathrm{eV} 185 \mathrm{keV}$ & ORELA $^{a}$ & Trans. at $78 \mathrm{~m}$ \\
\hline Pandey et al. [5] & $1 \mathrm{keV} 1,400 \mathrm{keV}$ & ORELA & Trans. at $78 \mathrm{~m}$ \\
\hline Guber et al. [6] & $100 \mathrm{eV} 90 \mathrm{keV}$ & GELINA $^{b}$ & Cap. at $58 \mathrm{~m}$ \\
\hline Guber et al. [6] & $100 \mathrm{eV} 2,200 \mathrm{keV}$ & GELINA & Cap. at $58 \mathrm{~m}$ \\
\hline Sobes et al. [7] & $0.01 \mathrm{eV} 0.1 \mathrm{eV}$ & MITR $^{c}$ & Trans. at $1.2 \mathrm{~m}$ \\
\hline
\end{tabular}

${ }^{a}$ Oak Ridge Electron Linear Accelerator.

${ }^{b}$ Geel Electron LINear Accelerator Facility.

${ }^{c}$ MIT Nuclear Research Reactor.

\section{Background}

A new resolved resonance region (RRR) evaluation for the two naturally occurring isotopes of copper- ${ }^{63} \mathrm{Cu}$ and ${ }^{65} \mathrm{Cu}$-was performed at Oak Ridge National Laboratory.

Copper is commonly used as a minor structural material in many fission power facilities. In fact, a search through the ICSBEP handbook reveals that copper is significant in 32 of the 586 evaluated criticality safety benchmarks and is found in 1,084 cases documented in ICSBEP. Copper is also an important structural component in Scandinavian spent fuel final disposal canisters.

Copper is an important heat sink material for fusion power reactors, and it is also used for diagnostics, microwave waveguides, and mirrors in the International Thermonuclear Experimental Reactor (ITER). The available nuclear data evaluations for stable ${ }^{63} \mathrm{Cu}$ and ${ }^{65} \mathrm{Cu}$ are not as consistent as required for accurate activation and particle transport calculations in fusion systems. Consistent, correct data evaluations are of high priority for applications to the International Fusion Materials Irradiation Facility (IFMIF), where the equipment must resist high-energy particle irradiation. As a prerequisite, the general-purpose neutron data files must include all information required for a wide spectrum of nuclear analyses, including simulations of neutrons and the assessment of the nuclear heating, damage, and gas production. The more accurate nuclear data are an important element of quality analyses for the design and optimization of all fusion related facilities including ITER and its planned successor reactor, the DEMOnstration Power Plant (DEMO), as well as the International Fusion Materials Irradiation Facility (IFMIF) neutron source.

The neutron cross section of the copper isotopes is also important to the astrophysics community. The two stable isotopes of copper lie along the beta-decay valley of stable isobars involved in the s process or slow-neutron-captureprocess. In the s process, successive neutron capture and then beta-decay reactions produce heavier isotopes. The sprocess occurs in stars and is responsible for the creation of approximately half of the stable isotopes heavier than iron, so it plays an important role in galactic chemical evolution. Improving copper evaluations will aid astrophysicists in reducing the uncertainty in their current models of the universe.

To address the nuclear criticality safety community's concerns regarding the performance of the copper isotopes in integral benchmarks, V. Sobes, L.C. Leal, et al. [4] re-evaluated the resolved resonance region of the two isotopes of copper based on the experimental data sets shown in Table 1. They extended the resolved resonance
Table 2. Collection of ICSBEP benchmarks used to test performance of resolved resonance region evaluations for ${ }^{63} \mathrm{Cu}$ and ${ }^{65} \mathrm{Cu}$.

\begin{tabular}{|c|c|}
\hline ICSBEP Benchmark Name & SAMINT usage \\
\hline IMF-20-03 & Used \\
\hline IMF-20-04 & Control \\
\hline IMF-20-05 & Control \\
\hline IMF-20-06 & Control \\
\hline IMF-20-07 & Control \\
\hline HMF-72-01 & Used \\
\hline HMF-72-03 & Control \\
\hline HMF-73-01 & Used \\
\hline HMF-84-06 & Used \\
\hline HMF-84-18 & Control \\
\hline HMF-85-01 & Used \\
\hline HMF-85-02 & Control \\
\hline HMF-85-04 & Control \\
\hline HMI-06-01 & Used \\
\hline HMI-06-02 & Used \\
\hline HMI-06-03 & Used \\
\hline HMI-06-04 & Used \\
\hline IMI-01-01 & Control \\
\hline IMI-01-02 & Control \\
\hline IMI-01-03 & Control \\
\hline IMI-01-04 & Control \\
\hline IMI-01-06 & Control \\
\hline IMI-01-07 & Control \\
\hline PMF-40-01 & Control \\
\hline
\end{tabular}

region of the two copper isotopes from $99.5 \mathrm{keV}$ to as far as the resolution of the experimental data allowed: $300 \mathrm{keV}$. This put resonance data into a larger portion of the intermediate and fast neutron spectrum criticality safety benchmarks. Furthermore, experimental capture cross section measurements were analyzed for the first time in the resonance evaluation of copper. Analyzing both the capture and the transmission experimental data sets allows for the capture-to-scattering ratio to be set correctly for the entire resolved resonance region.

There are at least twenty-four criticality safety benchmarks from the ICSBEP [3] available which demonstrable sensitivity of the benchmark quantity with respect to changes in the parameters of the resolved resonance region for the two isotopes of copper. The collective set of ICSBEP benchmarks used to test the performance of the updated copper evaluations is presented in Table 2 using the shorthand ICSBEP nomenclature.

\section{Methods}

Both the continuous-energy TSUNAMI-3D [8] module of the SCALE 6.2 Code System [9] and MCNP6 [10] can be used to produce energy-binned sensitivity coefficients of the integral system eigenvalue to different reaction cross sections based on continuous-energy physics. We define the relative sensitivity coefficient $S_{i j n}$ of the eigenvalue of system $i, k_{i}$, with respect to partial cross section $j$ in energy bin $n$ as

$$
S_{i j n}=\frac{\left\langle\sigma_{j}(E)\right\rangle_{n}}{k_{i}} \frac{\partial k_{i}}{\partial\left\langle\sigma_{j}(E)\right\rangle_{n}} .
$$




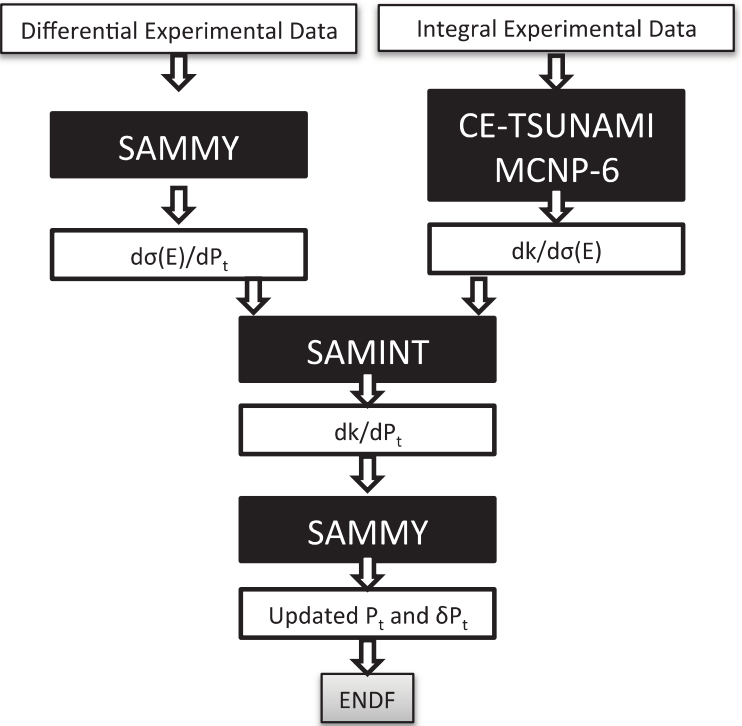

Figure 1. Work flow required to adjust nuclear data based on integral experimental data. The black boxes are codes, the white boxes are information, and the gray boxes are databases.

In the SAMMY Bayesian updating process, the derivative of the continuous-energy reaction cross section $j$ with respect to resonance parameter $t, P_{t}$, is needed and is therefore calculated by SAMMY. The symbol $P_{t}$ is a general symbol that stands for all resonance parameters $E_{\lambda}, \Gamma_{\gamma}, \Gamma_{n}, \Gamma_{f}$ etc. We define this derivative as $G_{j t}(E$, where

$$
G_{j t}(E)=\frac{\partial \sigma_{j}(E)}{\partial P_{t}} .
$$

A very fine energy mesh is used for the calculations, allowing the following two approximations:

$$
\sigma\left(E_{n}\right) \approx\left\langle\sigma_{j}(E)\right\rangle_{n}
$$

and

$$
G_{j t}\left(E_{n}\right)=\frac{\partial \sigma_{j}(E)}{\partial P_{t}} \approx \frac{\partial\left\langle\sigma_{j}(E)\right\rangle_{n}}{\partial P_{t}},
$$

where the variable $E_{n}$ is the middle of energy bin $n$. The sensitivity of the integral system eigenvalue $k_{i}$ to the resonance parameter $P_{t}$ is then

$$
\frac{\partial k_{i}}{\partial P_{t}}=\sum_{j} \sum_{n} \frac{k_{i}}{\sigma_{j}\left(E_{n}\right)} S_{i j n} G_{j t}\left(E_{n}\right) .
$$

SAMINT was created to carry out the summation in Eq. (5) and pass the resulting partial derivatives back to SAMMY. Consequently, SAMMY performs the same Bayesian updating procedure of nuclear data parameters, but it is based on the integral data experimental values (i.e., $k_{i}$ ) rather than the differential data experimental values (i.e., $\sigma_{j}(E)$. Figure 1 shows the work flow for using SAMINT.

\section{Results}

Extended runs of MCNP 6.1 [10] were used to calculate the sensitivity coefficients on the benchmark quantity (keigenvalue) to the capture and elastic scattering cross

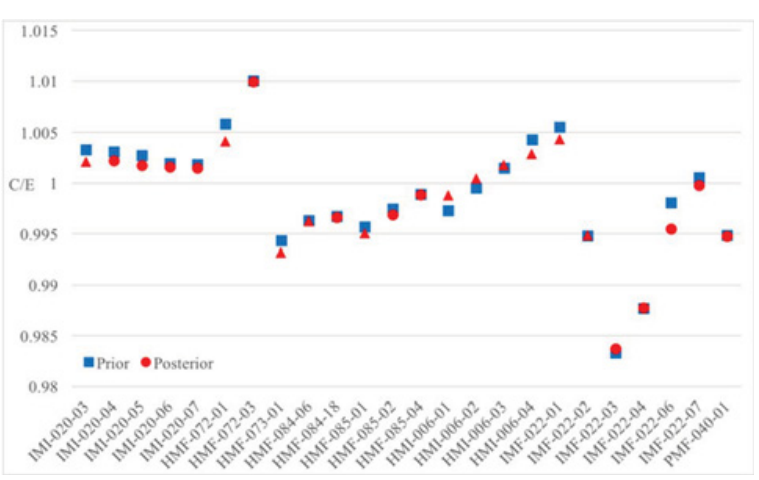

Figure 2. Results of the SAMINT adjustment methodology. The stochastic uncertainty of the Monte Carlo simulation was converged to well beyond the size of the markers. The integral experiments not used in the SAMINT adjustment process are labelled with a circle marker, while those used in the adjustment process are labelled with a triangle marker.

sections of the two isotopes of copper on an ultrafine energy mesh (Eq. (1)). Only the integral experiments labelled as Used in Table 2 were used to influence the adjustment of the resonance parameters. The other integral experiments, which are labelled as Control in Table 2, were not used to influence the adjustment of the resonance parameters; they were reserved to study the effect that the adjustment process will produce.

For the cross sections of the other isotopes in the models, the default ENDF/B-VII.1 cross sections distributed with MCNP were used, with the exception of ${ }^{1} \mathrm{H},{ }^{16} \mathrm{O},{ }^{56} \mathrm{Fe},{ }^{235} \mathrm{U},{ }^{238} \mathrm{U}$ and ${ }^{239} \mathrm{Pu}$, which were taken from the beta release of the Collaborative International Evaluated Library Organisation (CIELO) project [11].

Further, the SAMINT code was used to combine the computed sensitivity coefficients of the integral benchmarks with the calculated derivatives of the cross sections with respect to the resonance parameters (see Eq. (5)) to produce input files into the evaluation code SAMMY. The defining characteristic of the SAMINT approach is that in the following step, suggested resonance parameter adjustments from the integral experiments are combined using the GLLS mathematical framework with the resonance parameter adjustments based on the differential experimental data, thereby ensuring that the adjustments to the resonance parameter will not disrupt the fit of the experimental cross section measurements outside of statistical limitations.

Figure 2 presents the ratio of the calculated value for the benchmark quantity for each of the integral experiments to the respective experimentally measured value. The stochastic uncertainty of the Monte Carlo simulation was converged to be significantly smaller than the marker in Fig. 2. The integral experiments not used in the SAMINT adjustment process are labelled with a circle marker, while those used in the adjustment process are labelled with a triangle marker.

\section{Discussion}

Figure 2 shows that SAMINT is not a panacea; it does not automatically make all calculated benchmark results closer to their experimentally measured values. This is because the continuous-energy discrepancy for 
benchmarks containing copper can have supporting or conflicting messages. For example, consider the set of Intermediate enrichment - Metal fuel - Intermediate neutron spectrum (IMI) experiments that all calculate too high or the different High enrichment - Metal fuel - Fast neutron spectrum (HMF) experiments that all calculate too low. As a general indictor, the IMI experiments would suggest that an increase in the capture cross section is necessary, while the HMF experiments would suggest a decrease. The two sets are fairly consistent amongst themselves, while on a large scale, they are contradictory to each other. The mathematically rigorous approach of SAMINT makes the treatment of this inconsistency systematic. The consistent message within one group of benchmarks is often linked to the correlated nature of a series of integral experiments such as the IMI series. SAMINT does have the capability to statically treat correlations amongst all of the integral experiments when those are available. Since the IMI experiments are obviously correlated but those correlations are not well known, only one experiment from that series was used to perform the resonance parameter adjustment for this study. However, the effect of the positive correlation between the IMI series with respect to the cross sections of copper is evident, as all of the calculated values move in the same direction.

One of the more interesting results of the SAMINT adjustment is the improvement of the continuous-energy values for the HMI-006 experiments, as seen in the center of Fig. 2. All four of the experiments were included in making the adjustment because of their interesting, and well-documented trend across cases 01 to 04 . The drastic improvement in the continuous-energy value of those experiments compared to some others in the set can be justified by the fact that the reported experimental uncertainty on the benchmark value of those experiments is significantly lower than that of the other integral experiments included in this adjustment.

The last important discussion point is the strong dependence of the SAMINT adjustment process on cross sections used for all of the other isotopes needed to calculate the benchmark quantity for these integral experiments. Obviously, an error in the continuous-energy value caused by a poor cross section evaluation for one of the other isotopes used in the calculations will influence the results of the SAMINT adjustment process. However, the diversity of the integral experiments used somewhat guards from an error in the evaluation of one of the other isotopes driving the entire adjustment process. Further, cross section evaluation is a convergent process in which, with time, significant errors from cross section evaluations are eliminated, making minor adjustments with methods like SAMINT more relevant. Finally, the entirety of the SAMINT methodology is based on constraining the allowed changes to the resonance parameter by analyzing differential experimental data in parallel with integral experiments so that changes driven by integral experiments that would be contradictory to measured cross section values are not allowed.

This work was supported by the Department of Energy (DOE) Nuclear Criticality Safety Program, funded and managed by the National Nuclear Security Administration for DOE.

\section{References}

[1] V. Sobes, L. Leal, G. Arbanas, B. Forget, Nucl. Sci. \& Eng. 183(3), 347-355 (2016)

[2] N.M. Larson, Updated Users' Guide for SAMMY: Multi-level R-Matrix Fits to Neutron Data Using Bayes' Equations, United States Department of Energy (2003)

[3] B. Briggs, et al., International Handbook of Evaluated Criticality Safety Benchmark Experiments, Report NEA No. 7166 (2013)

[4] V. Sobes, L.C. Leal, K. Guber, B. Forget, S. Kopecky, P. Schillebeeckx, P. Siegler, Nuclear Data Sheets 118, 155-157 (2014)

[5] M.S. Pandey, J.B. Garg, J.A. Harvey, Phys. Rev. C, 15, I 2, 600 (1977)

[6] Personal communication with Klaus Guber (2011)

[7] V. Sobes, R. Macdonald, L. C. Leal, et al., Trans. Am. Nucl. Soc. 105 (2011)

[8] C.M. Perfetti, B.T. Rearden, Proc. of the 2013 Joint Int. Conf. on Supercomputing in Nuc. Appl. and Monte Carlo (2013)

[9] B.T. Rearden and M.A. Jessee, eds., "SCALE Code System," Oak Ridge National Laboratory report ORNL/TM-2005/39, Version 6.2 (2016). Available from Radiation Safety Information Computational Center as CCC-834

[10] J.T. Goorley, et al., Initial MCNP6 Release Overview - MCNP6 version 1.0, LA-UR-13-22934 (2013)

[11] M.B. Chadwick, et al., Nuclear Data Sheets 118, 125 (2014) 